\title{
Phosphorylated KDR can be located in the nucleus of neoplastic cells
}

\author{
Cristina Blazquez ${ }^{1}$, Nathan Cook $^{2}$, Kingsley Micklem³ ${ }^{3}$ Adrian L Harris ${ }^{4}$, Kevin C Gatter ${ }^{2}$, \\ Francesco Pezzella ${ }^{2}$
}

${ }^{1}$ Departamento de Bioquimica y Biologia Molecular I, Facultad de Biologia, Universidad Complutense de Madrid, Ciudad Universitaria, Madrid 28040, Espania; ${ }^{2}$ Cancer Research UK Tumour Pathology Group, Nuffield Department of Clinical Laboratory Sciences, John Radcliffe Hospital, Oxford, OX3 9DU, UK; ${ }^{3}$ Medical Informatics Unit, Nuffield Department of Clinical Laboratory Sciences, John Radcliffe Hospital, Oxford, OX3 9DU, UK; ${ }^{4}$ Cancer Research UK Molecular Oncology Laboratory, John Radcliffe Hospital, Oxford, OX3 9DU, UK

KDR (kinase insert domain receptor) phosphorylation induces several effects which lead eventually to cell proliferation and survival. The precise mechanisms by which KDR, once it is activated, communicates with the nucleus are starting to be understood but have not yet been completely unravelled. Two in vitro studies on animal cell lines reported in the literature have demonstrated that, following stimulation with VEGF, KDR is actually translocated within the nucleus. Our aim was to investigate whether this translocation occurs in human cells both in vitro and in vivo. Using laser scanning confocal microscopy, a variable nuclear localization of phosphorylated and total KDR in cell lines and tumour samples was found. In human neoplastic cell lines, hypoxic stimulation greatly increased the nuclear amount of total KDR but less so that of the phosphorylated form. Only after hypoxia and VEGF stimulation there was a comparably increased expression of phosphorylated and total KDR observed in the nuclei of these cells. We conclude that neoplastic cells show a variable expression of total and phosphorylated KDR in the nucleus. The precise functional meaning of nuclear location remains to be established.

Cell Research (2006) 16:93-98. doi:10.1038/sj.cr.7310012; published online 16 January 2006

Keywords: KDR, human neoplasma, hypoxia, nucleus

\section{Introduction}

Angiogenesis, the process of blood vessel formation from pre-existing vessels and vascular endothelial cells, is essential for normal growth and tissue repair, but is also critical in a wide variety of pathological conditions, such as diabetic retinopathy, arthritis, tumour growth and metastasis [1-3].

Vascular endothelial growth factor (VEGF) is essential for the development of the vascular system and acts as an endothelial cell-specific mitogen as well as a mediator of vascular growth $[4,5]$. Two high affinity receptors for

Correspondence: Francesco Pezzella

Tel: +44-01865-220497; Fax: +44-01865-222916;

E-mail: francesco.pezzella@cancer.org.uk

Received 25 May 2005; revised 14 Oct 2005; accepted 22 Oct 2005, published online 16 January 2006
VEGF, namely Flt-1 (VEFGR-1) and KDR (Flk-1/VEGFR2), have been identified [6-9]. These receptors belong to the tyrosine kinase receptor family. This family of receptors is characterised by the presence of seven immunoglobulin-like domains (Ig domains) in their extracellular ligand-binding part. Their intracellular domain in contrast comprises a single transmembrane domain and an intracellular tyrosine kinase domain split by a kinase insert that is important for substrate recognition [9]. All VEGFRs are expressed at an early stage during development and they display overlapping expression profiles, mostly confined to endothelial cells $[6,9]$.

KDR (VEGFR2) is a $230 \mathrm{kDa}$ protein which exerts its effect through activation of its intracellular tyrosine kinase domain. KDR can be phosphorylated on several tyrosine residues (Y801, Y951, Y996, Y1054, Y1059, Y1175 and Y1214) [10, 11]. Phosphorylation of the activation-loop tyrosine residues is required for activation of the catalytic 
activity of the KDR kinase [10]. KDR is expressed on endothelial cells. On the basis of ligand specificity, most functional VEGF cell signalling on endothelial cells described to date is mediated via KDR. This means that the major biological functions of VEGF, such as endothelial cell survival, proliferation and migration, increase of endothelial Nitrous Oxide (NO) and prostacycline production, increase of vascular permeability and promotion of mitogenic effects, are related to KDR mediated signalling $[12,13]$.

KDR is also expressed in haematopoietic stem cells, megakaryocytes, platelets and retinal cell progenitors [14-16]. KDR knock-out mice display embryonic lethality through their failure to produce endothelial and haematopoietic cells. Furthermore, KDR has been shown to be present in a number of neoplastic cell lines including melanoma, ovarian carcinoma, prostatic carcinoma [16] and in tumours, healing wounds and inflammatory sites [17].

Hypoxia is a major regulator of VEGF production under both physiological and pathological conditions. Regulation of VEGFR expression appears to be less straightforward. Experiments in mice have shown that both VEGFR-1 and KDR are induced under hypoxic conditions $[18,19]$. In endothelial cells lining the blood vessels of tumours VEGFR expression is also induced by hypoxia which causes the local concentration of VEGF to be increased [20].

It has been reported that there is a redistribution and internalisation inside the cytoplasm of KDR after phosphorylation [21]. Two other studies in vitro on bovine [20] and rat endothelium [22] have demonstrated that following stimulation with VEGF, KDR is actually translocated within the nucleus. However it is not known whether this translocation occurs in vivo and no studies have been carried out on human cells

An immunohistochemical study from our laboratory with anti-phosphorylated KDR antibodies showed that KDR can be expressed on normal cells other than endothelium and on neoplastic cells. It also showed the presence of what looked like nuclear KDR after staining with both anti phosphorylated and wild type antibodies [23]. These novel and somewhat surprising findings are potentially very important for understanding the function of KDR.

The aim of this study was to indicate whether total and/ or phosphorylated forms of KDR could be demonstrated inside the nuclei of human cells using laser scanning confocal microscopy.

\section{Material and methods}

\section{Cell culture}

The following cell lines were obtained from ATCC (American Type Culture Collection): MCF-7 (breast carcinoma), HeLa (carcinoma of the cervix) and HL60 (Acute Myeloid Leukaemia,
Promyelocytic). MCF-7 cells were cultured at $37^{\circ} \mathrm{C}$ and $5 \% \mathrm{CO}_{2}$ in E4 medium (GIBCO) containing 10\% fetal calf serum (FCS) in 75 $\mathrm{cm}^{2}$ flasks growing until 80-90\% of confluence. HeLa and HL60 cells were cultured at $37^{\circ} \mathrm{C}$ with $5 \% \mathrm{CO}_{2}$ in RPMI 1640 medium (GIBCO) containing $10 \%$ fetal calf serum (FCS) in $75 \mathrm{~cm}^{2}$ flasks growing to $80-90 \%$ confluence.

\section{Cell stimulation}

The HeLa cells were investigated not only in basal conditions but also after stimulation with hypoxia alone or hypoxia and VEGF 165.

Hypoxic stimulation: HeLa cells at approximately $70-80 \%$ confluence were transferred to a serum-free medium for $24 \mathrm{~h}$ and incubated for $16 \mathrm{~h}$ at $37^{\circ} \mathrm{C}$ with $0.1 \% \mathrm{O}_{2}$, fixed in PBS containing $10 \%$ of formalin, paraffin embedded and $5 \mathrm{~mm}$ sections were obtained.

Hypoxic and VEGF stimulation: HeLa cells at approximately 70 $80 \%$ confluence were transferred to a serum-free medium for $24 \mathrm{~h}$ and incubated for $16 \mathrm{~h}$ at $37^{\circ} \mathrm{C}$ with $0.1 \% \mathrm{O}_{2}$ before being stimulated with $50 \mathrm{ng} / \mathrm{ml}$ VEGF 165 (Astro Zeneca, R\&D Systems) for $8 \mathrm{~min}$. Cells were subsequently fixed in PBS with $10 \%$ formalin, paraffin embedded and $5 \mathrm{~mm}$ sections were obtained.

\section{Tumour samples}

Tumour tissues were obtained from the Department of Cellular Pathology, John Radcliffe Hospital, Oxford. The samples were formalin fixed, paraffin embedded and $5 \mathrm{~mm}$ sections were produced. Ethical approval for the use of the tissue was obtained.

Tumours studied included 5 of renal clear cell carcinomas, 4 of leukaemias ( 2 of acute myeloid and 2 of acute lymphoid), 6 of lymphomas ( 2 of mantle cell, 2 of peripheral T cell and 2 of small lymphocytic) and 4 of non- small cell lung cancers.

\section{Double immunofluorescence staining and laser scanning confocal microscopy}

To detect phosphorylated KDR we employed the monoclonal antibodies 34a and 126a produced in our laboratory and described elsewhere [23]. 34a recognises peptide 31 of KDR that contains the tyrosine residue Y1214, a potential Grb2 SH2 binding site that is related to an autophosphorylation site. 126a recognises peptide 25 of KDR containing the tyrosine residues Y1054/1059 located in the KDR activation loop.

We also used a polyclonal commercial antibody against phosphorylated KDR, Tyr996 (Cell Signaling Technology USA) that recognizes the peptide containing tyrosine 996 located in the tyrosine kinase insert loop.

Finally, we studied the localisation and expression of total KDR in cells using the commercial monoclonal antibody Flk-1 (A3) (Santa Cruz, USA) that recognizes the amino acid sequence corresponding to $1158-1345$ of the protein independently from its phosphorylation status.

Cell membrane was stained using the anti Epithelial Membrane Antigen (EMA) mouse monoclonal antibody E29 (DACOCYTOMATION).

Sections were deparaffinised and rehydrated. Antigen retrieval was carried out by immersing the slides in $10 \mathrm{mM}$ citrate at $\mathrm{pH} 6.0$ and boiling them for $3 \mathrm{~min}$. Slides were incubated for $1.5 \mathrm{~h}$ with the primary antibodies at the following dilutions: $34 \mathrm{a}(1: 20), 126 \mathrm{a}(1: 50)$ Tyr996 (1:50) and Flk-1 (A3) (1:50). After washing with TBS, the slides were incubated for $1 \mathrm{~h}$ with a mixture of the secondary Alexa 
Fluor 488 goat anti-mouse IgM (m chain) and Alexa Fluor 546 goat anti-mouse $\operatorname{IgG}_{1}(\mathrm{~g} 1)$ (1:400 for tumour samples, 1:100 for cell samples) (Molecular Probes Europe BV) in the case of monoclonal antibodies. A mixture of the secondary Alexa Fluor 488 goat antimouse $\operatorname{IgG}_{1}(\mathrm{~g} 1)$ and Alexa 546 goat anti-rabbit $\operatorname{IgG}(\mathrm{H}+\mathrm{L})(1: 400$ for tumour samples, 1:100 for cell samples) (Molecular Probes Europe BV) was used to detect the primary polyclonal commercial antibody.

All of the incubations were carried out at room temperature and in darkness. After washing with TBS, the sections were fixed with paraformaldehyde $1 \%$ for $10 \mathrm{~min}$, and mounted with DAKO's fluorescence mounting medium containing TOTO-3 iodide (1:500) (Molecular Probes Europe BV) to stain nuclei. Confocal fluorescent images were acquired using a ZEISS LSM510 microscope. Colour channels in the images were assigned as follows: Red-546nm excitation; Green-488nm excitation; Blue-633nm excitation.

\section{Results}

\section{pKDR expression in neoplastic cell lines}

Using confocal microscopy double immunofluorescence staining of MCF-7, HeLa and HL60 cells with 34a and 126a showed an internal distribution of the phosphorylated form of KDR with a predominant perinuclear localisation and a variable nuclear expression. With the commercial polyclonal anti-phosphorylated VEGF Receptor-2 (Tyr996), the fluorescence staining was stronger and more staining was located inside the nucleus.

Total KDR detected with the Flk-1 (A3) antibody showed a wide distribution inside the cell, as cytoplasmic, perinuclear and nuclear fluorescence staining was observed. Figure 1 illustrates the staining achieved on MCF-7 cells stained with Flk-1(A3), the anti total KDR antibody and the anti-phosphorylated KDR antibody 34a.

\section{pKDR expression in HeLa cells after VEGF and/or hypoxia treatment}

Hypoxic stimulation, with or without VEGF greatly increased the nuclear localization of total KDR but less so that of the phosphorylated form (Figure 2). The staining pattern of phosphorylated KDR was again mostly perinuclear with antibody 126a (Figure 2B) and 34a.

\section{pKDR expression in human tumours}

In the tumours a heterogeneous pattern of expression of KDR was observed using the monoclonal antibodies $34 \mathrm{a}$ and 126a. Overall the pattern of staining observed on the neoplastic cells from tissue sections was comparable to that seen on neoplastic cell lines (Figure 3).

Kidney tumours: Immunostaining with monoclonal antibodies $34 \mathrm{a}$ and $126 \mathrm{a}$ showed that $\mathrm{pKDR}$ was present on the membrane and within the cytoplasm and perinuclear regions. Some spots were also found inside the nucleus of malignant cells in some of the cases studied. Total KDR
A
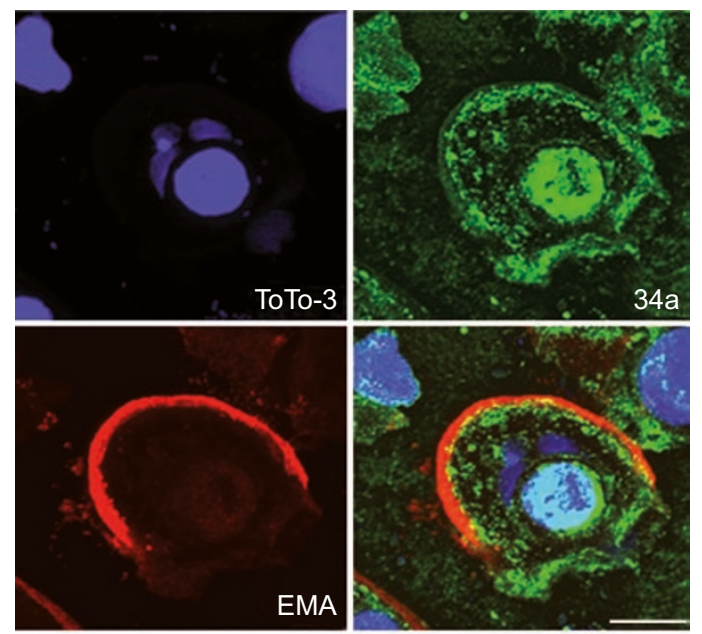

B
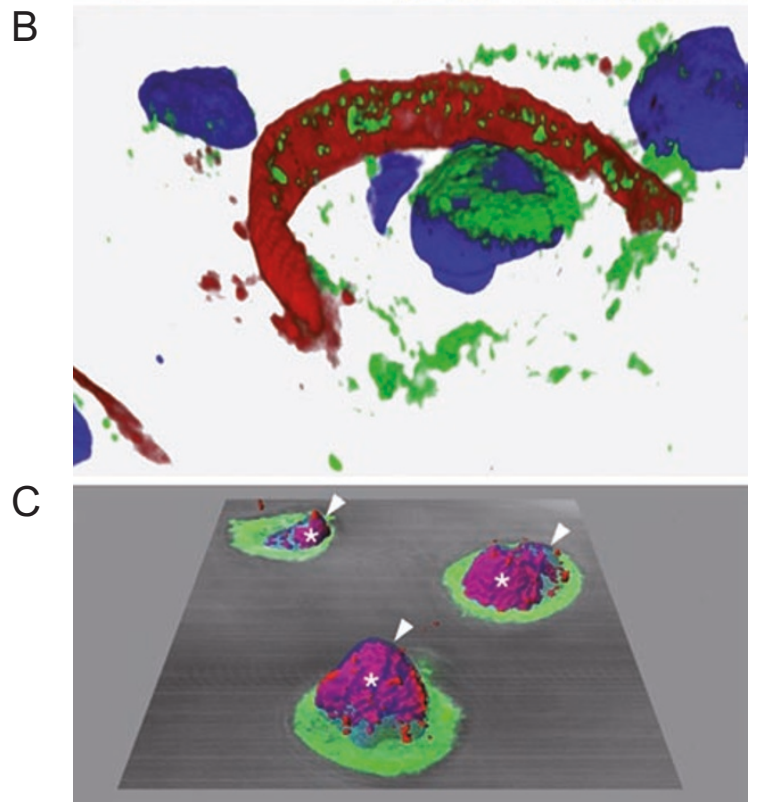

Figure 1 Confocal micrographs of MCF-7 cells. (A) Two dimension image of a single cell. Nuclear staining with TOTO-3 in blue, phosphorylated KDR stained with MoAb 34a in green, and cell membrane, stained for EMA, in red. Scale bar $=5 \mu \mathrm{m}$. (B) 3D reconstruction of the same cell shown in (A): $34 \mathrm{a}$ in green demonstrate the perinuclear phosphorylated KDR, the nucleus is stained in blue with TOTO-3 and the cell membrane in red. (C) 3D reconstruction cells stained with antibody 34a: An orthogonal plane intersecting 3 nuclei is shown as a two-dimensional DIC and 34a image. The cytoplasmic pKDR is represented in green. The TOTO-3 stained volume has been rendered as a blue solid with $60 \%$ transparency (arrowheads). Colocalized green and blue signals are rendered as a opaque red solid visible within the transparent nuclei representing the intranuclear pKDR (*).

[antibody Flk-1 (A3)] showed a similar pattern but with more staining localized to the nucleus of tumour cells.

Leukaemias: In the 2 AML cases, 34a staining showed 
both cytoplasmic and strong nuclear expression of pKDR in the neoplastic cells. Similarly, though more granular and less intense staining was found with antibody $126 \mathrm{a}$. In contrast, in the 2 cases of ALL pKDR expression was mostly cytoplasmic with only weak labelling in the nucleus. In all cases, total KDR [antibody Flk-1 (A3)] was mostly observed in the cytoplasm with only a slight expression in the nucleus.
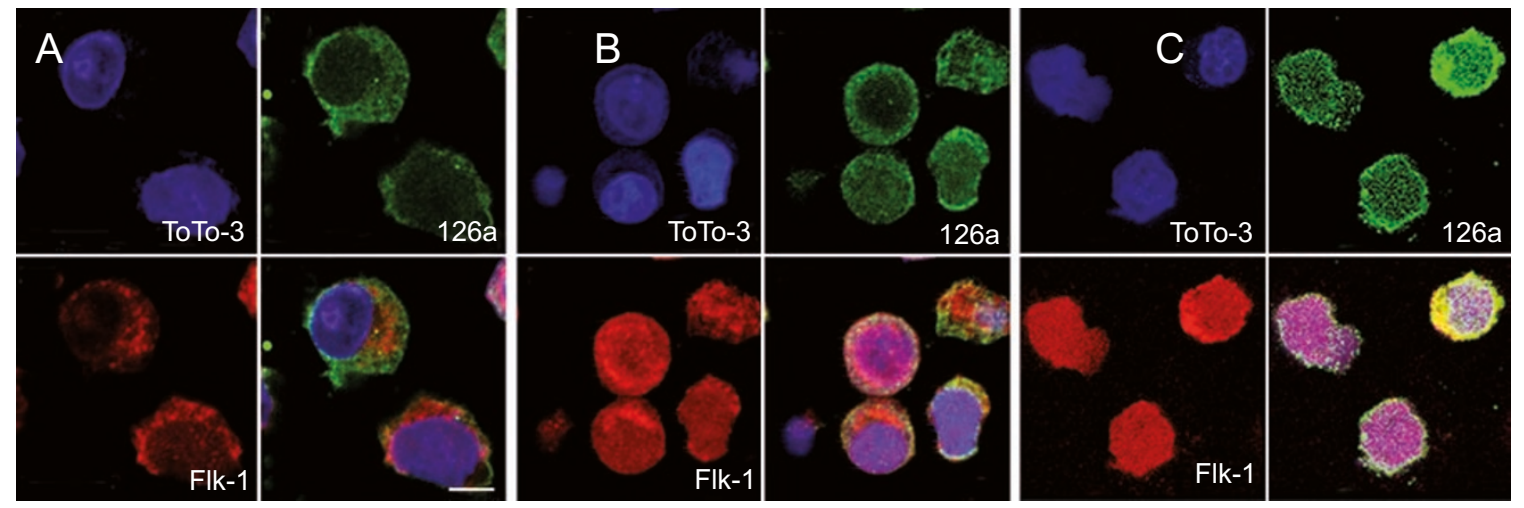

Figure 2 Fluorescence images of Hela cells by confocal microscopy. (A) native. (B) after stimulation with hypoxia. (C) stimulation with hypoxia plus VEGF $50 \mathrm{ng} / \mathrm{ml}$. Nuclear staining (with TOTO-3) in blue. MoAb 126a stains phosphorylated KDR in green. Flk1(A3) stains total KDR in red in all cases. Scale bar $=5 \mu \mathrm{m}$.
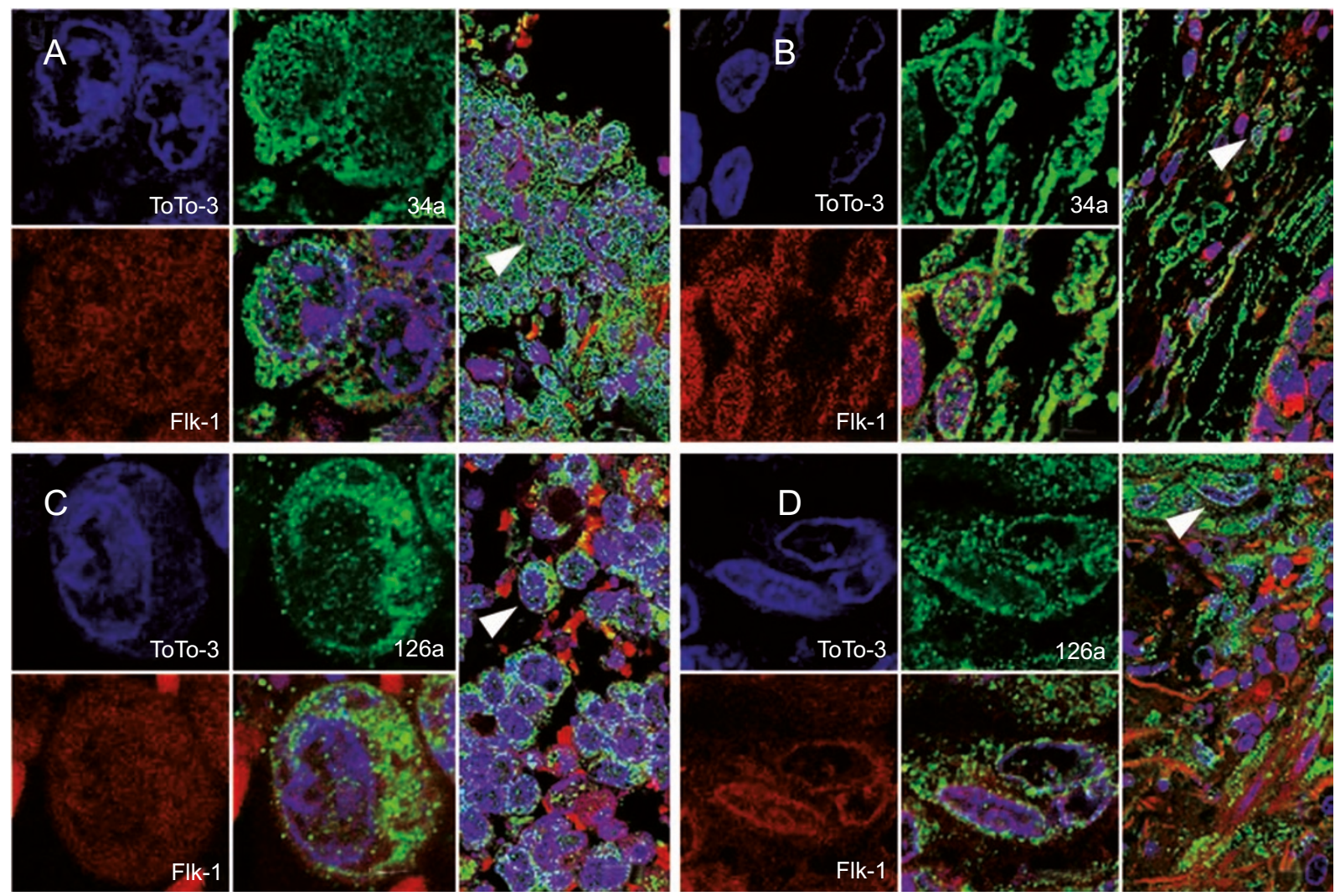

Figure 3 Fluorescence images of lung carcinomas by scanning confocal microscopy. 34a stains phosphorylated KDR in tumour cells (A) and in stromal cells (B) in green. 126a stains phosphorylated KDR in tumour cells (C) and in stromal cells (D) in green. Total KDR is stained with Flk-1(A3) in red. Cell nuclei are stained with TOTO-3 in blue. Short scale bar: $10 \mu \mathrm{m}$. Long scale bar=5 $\mu \mathrm{m}$. 
Lymphomas: 2 cases of peripheral $\mathrm{T}$ cell lymphoma showed cytoplasmic and strong perinuclear and nuclear staining for pKDR with both 34a and 126a. In contrast, in mantle cell lymphomas (MCL) and chronic lymphocytic leukaemia (B-CLL) the expression was only cytoplasmic and perinuclear. Total KDR [antibody Flk-1 (A3)] was present in the cytoplasm and the nucleus of all the lymphoma cases.

Lung, non-small cell carcinomas: $34 \mathrm{a}$ and 126a showed a strong granular fluorescence staining for pKDR in the membrane and cytoplasm of all 4 cases of lung cancer studied. Clear nuclear expression, although much weaker, was also recorded not only in the tumour cells but also in adjoining stromal cells. The Flk-1 (A3) antibody showed a widely distributed fluorescence staining throughout the tumour cells with total KDR being present in the membrane, cytoplasm and nucleus.

\section{Discussion}

KDR undergoes phosphorylation and induces several effects eventually leading to cell proliferation and survival. How KDR, once activated by VEGF, communicates with the nucleus is not yet completely understood. An earlier group of studies has shown that KDR phosphorylation leads to re-localisation in the cytoplasm but offers no proof of any nuclear localisation $[21,25,26]$. One in vitro study has shown that, following VEGF stimulation, KDR can be internalised within the cytoplasm [21]. Then, as shown in HUVEC cells after stimulation, KDR (but not Flt1) activation via multiple pathways such as MAPK/MSK-1 or C/p90RSK, can trigger CRE binding protein phosphorylation inside the nucleus [25].

Another pathway has been described in which non phosphorylated KDR (but not Flt-1) induces the translocation into the nucleus of STAT1 and STAT6. Indeed STAT1 is also known to co-precipitate with KDR. These data suggest, but do not prove, that KDR may be translocated inside the nucleus as well [26].

A number of other studies provide indirect evidence for a nuclear localization of $\mathrm{pKDR}$. Li et al showed that in bovine endothelial cells VEGF can be transiently translocated into the nuclei [19] and KDR, but not Flt1, was necessary for this to happen though there was no direct evidence of KDR itself within the nucleus.

Finally, two studies have demonstrated that KDR is translocated inside the nucleus [20-22] in association with endothelial Nitric Oxide Synthase (eNOS) by a process that may be mediated by a caveolar transport system [20]. None of this in vitro data can be automatically considered to be representative of what happens in vivo [28].
Our results conclusively demonstrate that in human cells, both in vitro and in vivo, phosphorylated KDR can be present inside the nuclei of normal and neoplastic cells. These results were obtained with each of the antibodies $34 \mathrm{a}$ and $126 \mathrm{a}$ - generated in our laboratory - and were confirmed on cell lines with an even clearer and stronger nuclear expression of phosphorylated KDR when the completely independent commercial antibody Tyr996 was used. This difference of intensity may be due to the fact that the antibodies recognise different tyrosine residues of the protein and also that the signal may be magnified by the polyclonal nature of the commercial antibody. Nevertheless the consistency of the nuclear staining is strong evidence in favour of its existence.

A number of representative tumours were selected in order to analyse the staining patterns of KDR expression. As might be expected, we obtained a variety of staining patterns finding membranous, cytoplasmic and nuclear expression of phosphorylated KDR that varied with the origin of the tumour cells. It is interesting to note that HL60 is a myeloid leukaemia cell line and, as in the case of myeloid leukaemia tissue, it showed strong and clear nuclear expression of activated KDR. This nuclear distribution might be related to the transcriptional activation of genes involved in the angiogenic cascade, but, at the moment, the precise function of this process is completely unknown. We also do not know the functional meaning of having phosphorylated KDR in the nucleus rather than in the cytoplasm.

However it is clear that nuclear translocation of the intracellular portion of membrane bound tyrosine kinase receptors upon activation has been described recently for other receptor molecules such as Erb B4, epidermal growth factor (EGF) receptor and FGFR-1 [29]. This suggests that this is an important mechanism involving their direct transcriptional regulatory activity in addition to roles in signal transduction. The mechanism whereby the membrane bound receptor tyrosine kinase, Erb B4, a member of the EGF receptor family, moves to the nucleus has been explored. This receptor undergoes proteolysis within the plasma membrane domain by regulated intramembranous proteolysis (RIP) and its intracellular portion moves to the nucleus where it may affect transcription of target genes $[29,30]$. However, prior to this, the ectodomain of the receptor is usually cleaved by a membrane associated metalloprotease. The intramembrane proteolysis described above is mediated by enzymes of the gamma secretase family which belongs to the group of presenilins or in some instances caspases [31].

Another possible mechanism of nuclear translocation is that the receptor undergoes secondary modifications, such as reduction in size possibly via intramembranous or 
cytoplasmic cleavage. These smaller receptor fragments may then relocate either alone or in concert with other molecules such as STAT-1. The role of such relocation can only be speculated upon. It may well be that these fragments have a direct transcriptional effect in tumour cells. This is an exciting possibility because if proven it should be possible to combine an anti-KDR small molecule inhibitor with conventional tumour treatment.

In conclusion, neoplastic cells showed a variable expression pattern of phosphorylated KDR between nucleus and cytoplasm. The functional meaning of these different intracellular locations remains to be established.

\section{Acknowledgements}

This work was funded by Cancer Research UK. Cristina Blazquez was supported by a grant from the Federation of European Biochemical Societies (FEBS).

\section{References}

1 Carmeliet P, Jain RK. Angiogenesis in cancer and other diseases. Nature 2000; 407:249-57.

2 Yancopoulos GD, Davis S, Gale NW, et al. Vascular-specific growth factors and blood vessel formation. Nature 2000; 407:242-8.

3 Fidler IJ. Regulation of neoplastic angiogenesis. J Natl Cancer Inst Monogr 2001; 28:10-4.

4 Ferrara N. VEGF: an update on biological and therapeutic aspects. Curr Opin Biotechnol 2000; 11:617-24.

5 Ferrara N. Role of vascular endothelial growth factor in regulation of physiological angiogenesis. Am J Physiol Cell Physiol 2001; 280:C1358-66.

6 de Vries C, Escobedo JA, Ueno H. et al. The fms-like tyrosine kinase, a receptor for vascular endothelial growth factor. Science 1992; 255:989-91.

7 Lee J, Gray A, Yuan J. et al. Vascular endothelial growth factor-related protein: a ligand and specific activator of the tyrosine kinase receptor Flt4. Proc Natl Acad Sci U S A 1996; 93:198892.

8 Terman BI, Dougher-Vermazen M, Carrion ME, et al. Identification of the KDR tyrosine kinase as a receptor for vascular endothelial cell growth factor. Biochem Biophys Res Commun 1992; 187:1579-86.

9 Soker S, Takashima S, Miao HQ, Neufeld G, Klagsbrun M. Neuropilin-1 is expressed by endothelial and tumor cells as an isoform-specific receptor for vascular endothelial growth factor. Cell 1998; 92:735-45.

10 Petrova TV, Makinen T, Alitalo K. Signaling via vascular endothelial growth factor receptors. Exp Cell Res 1999; 253:11730 .

11 Takahashi T, Yamaguchi S, Chida K, Shibuya M. A single autophosphorylation site on KDR/Flk-1 is essential for VEGFA-dependent activation of PLC-gamma and DNA synthesis in vascular endothelial cells. EMBO J 2001; 20:2768-78.

12 Brown LF, Detmar M, Claffey K, et al. Vascular permeability factor/vascular endothelial growth factor: a multifunctional angiogenic cytokine. Exs 1997; 79:233-69.

13 Zachary I, Gliki G. Signaling transduction mechanisms mediating biological actions of the vascular endothelial growth factor family. Cardiovasc Res 2001; 49:568-81.

14 Yang K, Cepko CL. Flk-1, a receptor for vascular endothelial growth factor VEGF, is expressed by retinal progenitor cells. J Neurosci 1996; 16:6089-99.

15 Ziegler BL, Valtieri M, Porada GA, et al. KDR receptor: a key marker defining hematopoietic stem cells. Science 1999; 285:1553-8.

16 Kendall RL, Rutledge RZ, Mao X, et al. Vascular endothelial growth factor receptor KDR tyrosine kinase activity is increased by autophosphorylation of two activation loop tyrosine residues. J Biol Chem 1999; 274:6453-60.

17 Tuder RM, Flook BE, Voelkel NF. Increased gene expression for VEGF and the VEGF receptors KDR/Flk and Flt in lungs exposed to acute or to chronic hypoxia. Modulation of gene expression by nitric oxide. J Clin Invest 1995; 95:1798-807.

18 Brekken RA, Thorpe PE. VEGF-VEGF receptor complexes as markers of tumor vascular endothelium. J Control Release 2001; 74:173-81.

19 Li W, Keller G. VEGF nuclear accumulation correlates with phenotypical changes in endothelial cells. J Cell Sci 2000; 113(Pt 9):1525-34.

20 Feng Y, Venema VJ, Venema RC, Tsai N, Caldwell RB. VEGF induces nuclear translocation of Flk-1/KDR, endothelial nitric oxide synthase, and caveolin-1 in vascular endothelial cells. Biochem Biophys Res Commun 1999; 256:192-7.

21 Dougher M, Terman BI. Autophosphorylation of KDR in the kinase domain is required for maximal VEGF-stimulated kinase activity and receptor internalization. Oncogene 1999; 18:161927.

22 Thuringer D, Maulon L, Frelin C. Rapid transactivation of the vascular endothelial growth factor receptor KDR/Flk-1 by the bradykinin B2 receptor contributes to endothelial nitric-oxide synthase activation in cardiac capillary endothelial cells. J Biol Chem 2002; 277:2028-32.

23 Stewart M, Turley H, Cook N, et al. The angiogenic receptor KDR is widely distributed in human tissues and tumours and relocates intracellularly on phosphorylation. An immunohistochemical study. Histopathology 2003; 43:33-9.

24 Risau W. Mechanisms of angiogenesis. Nature 1997; 386:6714.

25 Mayo LD, Kessler KM, Pincheira R, Warren RS, Donner DB. Vascular endothelial cell growth factor activates CRE-binding protein by signaling through the KDR receptor tyrosine kinase. J Biol Chem 2001; 276:25184-9.

26 Bartoli M, Gu X, Tsai NT, et al. Vascular endothelial growth factor activates STAT proteins in aortic endothelial cells. J Biol Chem 2000; 275:33189-92.

27 Valter MM, Hugel A, Huang HJ. et al. Expression of the Ets-1 transcription factor in human astrocytomas is associated with Fms-like tyrosine kinase-1 Flt-1/vascular endothelial growth factor receptor-1 synthesis and neoangiogenesis. Cancer Res 1999; 59:5608-14.

28 Mukhopadhyay D, Nagy JA, Manseau EJ, Dvorak HF. Vascular permeability factor/vascular endothelial growth factor-mediated signaling in mouse mesentery vascular endothelium. Cancer Res 1998; 58:1278-84.

29 Ni CY, Murphy MP, Golde TE, Carpenter G. Gamma secretase cleavage and nuclear localisation of ERB-B4 receptor tyrosine kinase. Science 2001; 294:2179-81.

30 Heldin CH, Ericsson J. RIPping tyrosine kinase receptors apart. Science 2001; 294:2111-3.

31 Strooper BD, Annaert W. Presenilins and the intramembrane proteolysis of proteins, facts and fiction. Nat Cell Biol 2001; 3: E221-5.

Edited by Shisan (Bob) Bao 